\title{
Behavioral and Functional Neuroimaging Evidence for Prefrontal Dysfunction in Methamphetamine-Dependent Subjects
}

\author{
Martin P. Paulus, M.D., Nikki E. Hozack, Blanca E. Zauscher, B.S., Lawrence Frank, Ph.D., \\ Gregory G. Brown, Ph.D., David L. Braff, B.A., M.D., and Marc A. Schuckit, M.D
}

Stimulant-dependent subjects show dysfunctions in decision-making similar to those seen in subjects with ventromedial prefrontal cortex lesions. Studies of drug craving, reward association, and decision-making have implicated dysfunctions of the dorsolateral and orbitofrontal cortex as a key neural substrate in subjects with stimulant dependence. Here, a functional magnetic resonance imaging (fMRI) study was carried out to determine the relationship between decision-making dysfunction and neural activation in different prefrontal areas. This investigation tested the behavioral hypothesis that methamphetamine-dependent subjects in early sustained remission show decision-making dysfunctions that are consistent with an increased reliance on stimulus-contingent response selection. It was hypothesized that these decision-making dysfunctions are due to differences in task-related activation in the dorsolateral and ventromedial prefrontal cortex. Ten methamphetamine-dependent subjects were compared with ten age- and education-matched controls performing a two- choice prediction task and a two-choice response task during a fMRI session. Response bias, latency, and mutual information measures assessing the underlying strategies of the decision-making sequences were obtained. First, methamphetamine-dependent subjects were more influenced by the immediately preceding outcome during the two-choice prediction task relative to normal comparison subjects. Second, methamphetamine-dependent subjects activated less dorsolateral prefrontal cortex (BA 9) and failed to activate ventromedial cortex $(B A 10,11)$ during the two-choice prediction task compared with the two-choice response task. These results support the basic hypothesis that stimulant-dependent subjects exhibit fundamental cognitive deficits during decision-making that are consistent with both orbitofrontal and dorsolateral prefrontal dysfunction.

[Neuropsychopharmacology 26:53-63, 2002]

(C) 2001 American College of Neuropsychopharmacology.

Published by Elsevier Science Inc.
From the Laboratory of Biological Dynamics and Theoretical Medicine, University of California San Diego, San Diego, CA (MPP, NEH, BEZ, LF); Department of Psychiatry, University of California San Diego, San Diego, CA (MPP, GGB, DLB, MAS); and Department of Radiology, University of California San Diego, San Diego, CA (LF); and San Diego Veterans Administration Medical Center, San Diego, CA (MPP, LF, GGB).

Address correspondence to: Martin P Paulus, Department of Psychiatry, UCSD, La Jolla CA 92093-0603; E-mail: martin@mag. ucsd.edu.

Received March 9, 2001; revised June 21, 2001; accepted July 18, 2001.

Online publication: 7/19/01 at www.acnp.org/citations/Npp 071901153.
The compulsive and nonadaptive nature of drug-taking behavior in substance-dependent subjects has led several investigators to hypothesize that subjects with substance dependence show a dysregulation of ventromedial or orbitofrontal cortex (London et al. 2000; Rolls 2000; Volkow and Fowler 2000). Disruption of the orbitofrontal cortex via the striato-thalamo-orbitofrontal neural systems loop, which is critical for the assessment of stimulus-reward relationships, has been proposed as a key neural substrate underlying the neural systems dysregulation in substance dependence (Volkow and 
Fowler 2000). Specifically, the orbitofrontal cortex maintains a detailed representation of rewarding stimuli and updates the current reward value of a stimulus continually (O'Doherty et al. 2000). Therefore, the orbitofrontal cortex is critical for rapid stimulus-reinforcement association learning and the correction of these associations when reinforcement contingencies change (Rolls 2000). The combination of dorsolateral and orbitofrontal cortex dysregulation in substance-dependent subjects may affect three distinct behavioral functions (London et al. 2000): first, expectancy, which is based on the predictions of reward and observed probabilities of reinforcement with a stimulus; second, compulsion, which involves the repetitive application of a behavioral strategy despite the lack of association of reward with the stimulus; third, decision-making, which involves the balancing of expectations with stimulusassociated rewards or reinforcing probabilities.

Decision-making includes several cognitive and noncognitive processes, such as attention, working memory (Bechara et al. 1994), contingency approximation (Tversky et al. 1988; Elliott and Dolan 1998), hypothesis testing (Elliott and Dolan 1998), rule generation (Seale and Rapoport 1997), impulsivity (Green et al. 1999; Monterosso and Ainslie 1999) and risk-taking (Rahman et al. 1999; Rogers et al. 1999a). The integrity of the orbitofrontal cortex is important for decision-making when the outcome is uncertain but some responses are associated with a better long-term outcome than others. Specifically, subjects with orbitofrontal cortex lesions perform poorly when asked to select advantageous responses and avoid disadvantageous responses (Bechara et al. 1994). Other decision-making tasks provide the subject with explicit information for each trial about the likelihood of beneficial or adverse outcomes and measure the degree to which subjects use this information to select their responses (Rogers et al. 1999a). We have further developed an experimental decision-making task in which subjects do not know a-priori which action is associated with the best outcome (Paulus et al. 1994, 1996). Moreover, each response has a similar probability to have a beneficial or adverse outcome. Previous results from behavioral experiments show that even though the reinforcement of each response is random, the sequence characteristics of the subject's responses are not (Paulus 1997). Thus, this task allows one to measure the degree to which past stimuli, past responses, or the combination of stimuli and responses influence the current response selection and helps to quantify strategies that underly decision-making in the presence of uncertainty.

Based on previous results of decision-making studies in subjects with substance dependence, which showed that decision-making was more contingent on immediate gains than long-term outcomes (Grant et al. 2000), this experiment tested the hypothesis that methamphet- amine-dependent subjects during early sustained remission show altered decision-making rules. Specifically, it was hypothesized that if methamphetaminedependent subjects are supersensitive to immediate gains, then response selection should be more predictable based on the preceding relationship between stimulus and response and should depend less on a self-generated, possibly long-range, response strategy. Moreover, if activation of ventromedial and dorsolateral prefrontal cortex is critical for the normal decision-making process (Elliott and Dolan 1998) and if the decision-making rules are altered in methamphetamine-dependent subjects, it was hypothesized that methamphetamine-dependent subjects show an altered level of decision-makingrelated activation in both orbitofrontal and dorsolateral prefrontal cortex. To test these hypotheses, we conducted a functional magnetic resonance imaging (fMRI) study using the two-choice prediction task with a group of methamphetamine-dependent subjects in early remission and age- and education- matched comparison subjects. Mutual information measures were used to determine the degree to which response selection was predicted by the previous stimulus-response association or the previous response. Both a voxel-wise comparison and a region of interest analysis were used to determine taskrelated activation differences in methamphetamine-dependent and normal comparison subjects.

\section{METHODS}

\section{Subjects}

Ten subjects with an average age of $41.1 \pm 2.4$ years (range 31-54) and an average education level of $13.6 \pm$ 0.35 years (range 12-15.5) meeting current stimulantdependence criteria according to the DSM-IV (American Psychiatric Association 1994) were recruited from the Alcohol and Drug Treatment Program (ADTP) at the San Diego Veterans Affairs Medical Center. These methamphetamine-dependent subjects had entered voluntarily a 28-day inpatient ADTP. All subjects were randomly screened for the presence of drugs, which was mandatory for the participation in this program. These subjects did not have serious medical conditions, did not exhibit significant affective, anxious, or cognitive symptoms, and had participated in outpatient groups before entering the inpatient program. None of these subjects were taking psychotropic medications at time of testing. Three of the 10 subjects also fulfilled criteria for current cannabis abuse but not dependence. The individual subjects' characteristics are listed in Table 1 . The behavioral and functional neuroimaging data from these subjects were compared with normal comparison subjects that were matched by age (mean $42.30 \pm$ 1.9 years, range $28-50$ ) and education (mean $14.5 \pm 0.43$ years, range 12-16). A subgroup of the normal compari- 
son subjects' data has been presented elsewhere (Paulus et al. 2001).

Once a subject agreed to be contacted and gave informed consent, a structured clinical interview for DSM IV diagnosis (SCID-P) (Spitzer et al. 1992) and the antisocial personality disorder (ASPD) segment of the SCID II for personality disorders were conducted. Subjects with a major depressive disorder, bipolar, schizophrenic, post-traumatic stress, panic, or obsessive-compulsive disorder or ASPD as well as subjects exhibiting current signs of withdrawal as indicated by the presence of at least two DSM IV withdrawal signs were excluded from the study. Subject with nonremovable materials that respond to high magnetic fields, for example, metal fragments, were also excluded. At the time of testing, these subjects had been abstinent from methamphetamine for an average of $22.4 \pm 3.5$ days (range 6-46 days), and urine toxicology revealed no evidence of cannabis, stimulants, sedative-hypnotics, cocaine, or Phenycydidine (PCP).

\section{Task}

The two-choice prediction task, which is used to determine the response characteristics in decision-making situations with uncertain outcome, has been described in detail elsewhere (Paulus 1997). Briefly, on a computer screen, a house is shown in the center with two people: one on the left and one on the right of the house. For the two-choice prediction task, the subject is told that the task is to predict whether a car will come by on the left or right side to pick up the person on the computer screen. The subject has to make a decision (pressing the left or right button) and is shown the car after pressing the button for $300 \mathrm{msec}$. If the selected response is "correct," that is, reinforced, the person of the selected side crosses over to the car; otherwise, the person moves halfway across the screen and then returns to the center of the screen. The reinforcement schedule is determined a priori such that $50 \%$ of the responses will be reinforced, as if they were "correct" predictions. For the two-choice response task, the subject is told that the task is to press the button on the same side that the car is shown on the screen (i.e., left or right). The duration of each trial depends on the time between presentation of the initial situation and the selection of the response. Therefore, the number of trials per experimental block depends on the subject's average latency to select a response during a trial block. The key difference between these two tasks is that during the two-choice prediction task, the subject does not know the correct response in advance. The only information that may guide the selection of the current response is the sequence of previous responses and outcomes. In comparison, during the two-choice response task, the subject knows the correct answer before selecting a response, and the current button press does not depend on the previous responses.

\section{Behavioral Measures}

The following variables were recorded for the twochoice prediction and the two-choice response tasks: (1) the response selected by the subject (left or right); (2) the computer selected response (left or right); and (3) the latency to select a response (time from the presentation of the current situation to button press). Based on these variables, the strategies of decision-making in the presence of uncertainty were assessed by two sets of measures: (1) general response biases: the number of left versus right responses or stay (a left response followed by left response) versus switch responses (left followed

Table 1. Clinical Characteristics of Methamphetamine-Dependent Subjects

\begin{tabular}{|c|c|c|c|c|c|c|}
\hline Subject & Age & Education & Race & $\begin{array}{c}\text { Use } \\
\text { (years) }\end{array}$ & $\begin{array}{c}\text { Sobriety } \\
\text { (days) }\end{array}$ & Comments \\
\hline 182 & 52 & 12 & $\mathrm{C}$ & 32 & 26 & \multirow{8}{*}{$\begin{array}{l}\text { THC abuse } \\
\text { (current) } \\
\text { THC abuse } \\
(1981-1984)\end{array}$} \\
\hline 360 & 49 & 12 & $\mathrm{C}$ & 19 & 11 & \\
\hline 490 & 38 & 14 & $\mathrm{C}$ & 18 & 25 & \\
\hline 491 & 42 & 13.5 & $\mathrm{C}$ & 22 & 46 & \\
\hline 1027 & 37 & 15.5 & $\mathrm{H}$ & 12 & 14 & \\
\hline 1228 & 35 & 12.5 & $\mathrm{C}$ & 17 & 19 & \\
\hline 1229 & 40 & 14.5 & $\mathrm{C}$ & 18 & 25 & \\
\hline 1230 & 54 & 14 & $\mathrm{C}$ & 33 & 27 & \\
\hline 1894 & 31 & 13 & B & 13 & 6 & \multirow[t]{3}{*}{$\begin{array}{l}\text { THC abuse } \\
\text { (current) }\end{array}$} \\
\hline 1911 & 46 & 12 & $\mathrm{C}$ & 15 & 10 & \\
\hline 2742 & 35 & 12 & $\mathrm{O}$ & 17 & 22 & \\
\hline
\end{tabular}

B, Black; C, Caucasian; H, Hispanic; O, other. All subjects were medication-free at time of testing; no other substance was present. 
by right response); and (2) the degree to which the current response is determined by the previous response, the previous stimulus, or a combination of both, which is quantified by mutual information measures. Mutual information functions (Herzel and Grosse 1997) are based on the logarithmic likelihood ratio between the observed and the expected frequencies of an event. These functions measure in units of bits the degree to which two events co-occur more often than by chance. In addition, the conditional probability of using winstay and lose-shift were obtained, i.e., the probability of selecting the same response or shifting to the alternate response if the previous response resulted in a "correct" or "incorrect" prediction. These measures allows one to determine whether the degree to which subjects used win-stay/lose-shift above chance level was due to an increased use of win-stay or lose-shift responses.

\section{fMRI Protocol and Image Analysis Pathway}

Magnetic resonance images were obtained using a 1.5 Tesla whole-body system (Siemens, Erlangen). Anatomical T1-weighted images of the whole brain (magnetization prepared rapid gradient echo (MPRAGE) resonance time $(\mathrm{TR})=11.4 \mathrm{~ms}$, echo time $(\mathrm{TE})=4.4 \mathrm{~ms}$, flip angle $=$ $10^{\circ}$, field of view $(\mathrm{FOV})=256 \times 256,1 \mathrm{~mm}^{3}$ voxels) were obtained sagitally to identify the anterior/posterior commissure, to co-register the functional image, and to transform the images into Talairach space (Talairach and Tournoux 1988). Thirty-two slices of T2-weighted images were obtained in the transverse plane using echoplanar imaging (slice acquisition interval $=60 \mathrm{~ms}$, $\mathrm{TE}=40 \mathrm{~ms}$, flip angle $=90^{\circ}, 64 \times 64$ pixel, $\mathrm{FOV}=220 \times$ $220 \mathrm{~mm}, 3.43 \mathrm{~mm}^{2}$ in-plane resolution, 3-mm slice thickness) with a repetition time (TR) of $3000 \mathrm{msec}$ for 112 repetitions. The 3-mm slice thickness greatly reduces the echoplanar signal reduction in the orbitofrontal areas.

The task was presented to the subjects using an liquid crystal display (LCD) projector, back-projected onto a screen at the subjects' feet, which could be seen via a mirror attached to the head coil. Subjects requiring corrective lenses were provided with a pair of plasticframed lenses that approximated their degree of correction. Motor responses were made using a fiber-optic button box. The two-choice prediction task ("on" condition) and the two-choice response task ("off" condition) were presented in an "off-on" repeated block-design. Each block lasted $30 \mathrm{~s}$ or 10 TRs. The entire experiment lasted 11 alternating "off-on" blocks, starting and ending with an "off" condition.

Three fMRI analysis pathways were used to determine (1) whether methamphetamine-dependent subjects show task-related activation differences relative to comparison subjects, (2) whether the magnitude of activation in task-related regions of interest, that is, areas that are activated during the two-choice prediction task in both groups, differs between methamphetaminedependent and normal comparison subjects, and (3) whether the degree of activation is related to clinical parameters. First, an fMRI block design was used to determine which neural substrates showed task-related activation (i.e., activated during the two-choice prediction task relative to the two-choice response task). Specifically, 112 whole-brain images (32 3-mm slices) were collected every $3 \mathrm{~s}$. After discarding the first two acquisitions, the remaining 110 functional brain images were divided into 11 trial blocks, each trial block lasting $30 \mathrm{~s}$ and consisting of 10 repetitions. All image processing was done using the Analysis of Functional Neuroimages (AFNI) software package (Cox 1996). Echoplanar images were co-registered using a 3D co-registration algorithm to the echoplanar image that resulted in the smallest amount of image translation and rotation relative to all other images. A regression analysis was performed with a 0-1 boxcar reference function, which was shifted 0-3 TRs to account for the hemodynamic response, to predict the fluctuation in the linearly detrended, non-normalized, echoplanar image intensities as measured by a voxelwise regression or fit-coefficient (Cohen 1997). A Gaussian filter with full width half maximum (FWHM) $3.4 \mathrm{~mm}$ was applied to the regression coefficient image to account for individual variations of the anatomical landmarks. Regression coefficient images of each subject were translated to Talairach coordinates. Labels for brain activation foci were obtained in Talairach coordinates using the Talairach Demon software, which provides accuracy similar to that of neuroanatomical experts (Lancaster et al. 2000).

\section{Statistical Analysis}

A mixed model analysis of variance (ANOVA) was used to analyze the behavioral measures. Specifically, one between-subjects factor (methamphetamine-dependent subjects versus controls) and two within-subjects factors (block: two-choice prediction task or two-choice response task; repetition: five repetitions of the block) were used to determine whether the behavior measures differed across groups and across the two task conditions. The block-effect tests for behavioral differences across task conditions. The repetition effect assesses whether subjects engage in different decision-making behavior across trial blocks. To adjust the degrees of freedom for the correlations in within-subjects designs (violations of sphericity), Greenhouse-Geisser (GG) corrections were applied.

A mixed model ANOVA (group = fixed factor, subjects $=$ random factor) was conducted with the Tailarach fit-coefficient image as the dependent measure to determine group differences between methamphetamine-dependent and normal comparison subjects. To 
control for false-positive regions of activation for the main effect of group (F-map) and the individual group effects (t-maps), the activation significance threshold was adjusted based on a Monte-Carlo simulation of filtered voxels' activation patterns (Forman et al. 1995). The images shown represent volume-thresholded $F$ or t-maps. Second, both methamphetamine-dependent and comparison subjects were pooled to determine common regions of task-related activation. For each region of task-related activation, an average fit coefficient was obtained for each subject and the region of interest (ROI) specific fit coefficient was used in planned t-tests to compare the degree of task-related activation in methamphetamine-dependent subjects relative to comparison subjects. Third, ROI specific fit coefficient was used as an independent variable in a stepwise regression analysis to predict years of methamphetamine use and duration of sobriety. Stepwise regression analyses were conducted by setting the threshold for entering a variable into the regression to $p<.05$ and a removal of a variable to $p>$.1. All behavioral statistical analyses were carried out using statistical package for the Social Sciences (SPSS) (Norusis 1990).

\section{RESULTS}

\section{Behavioral Measures}

Methamphetamine-dependent subjects made $35.2 \pm$ 1.25 responses (controls, $34.7 \pm 1.25$ ) during a choice response task trial block. These subjects selected the right response $51 \pm 0.8 \%$ (controls, $50.3 \pm 0.8 \%$ ) of all trials, and switched responses $48.2 \pm 0.5 \%$ (controls, $49.6 \pm$ $0.5 \%$ ) of the time during the two-choice response task trial block and thus showed no simple response bias. During the two-choice prediction task, methamphetamine-dependent subjects made $35.5 \pm 2.8$ responses (controls, $34.2 \pm 2.8$ ), selected the right response $49.7 \pm$ $1.7 \%$ (controls, $48.2 \pm-1.2 \%$ ) of the time, and switched responses $48.4 \pm 2.2 \%$ (controls, $47.7 \pm 2.2 \%$ ) of the time. Methamphetamine-dependent subjects made $4.5 \pm$ 1.3 (controls: $1.8 \pm 1.3$ ) errors per trial block during the two-choice response task. The ANOVA revealed that the basic response bias measures, that is, response number, left versus right response, and response switching versus response stay, did not differ across task conditions [response number, $\mathrm{F}_{\mathrm{GG}}(1,18)=0.01$, not significant (NS); right response, $\mathrm{F}_{\mathrm{GG}}(1,18)=2.9$, NS; response switching, $\mathrm{F}_{\mathrm{GG}}(1,18)=0.36$, NS] or across groups [response number, $\mathrm{F}(1,18)=0.10$, NS; right response, $\mathrm{F}(1,18)=0.46$, NS; response switching, $\mathrm{F}(1,18)=0.05$, NS]. In addition, the number of errors per trial block did not differ significantly between methamphetaminedependent and normal comparison subjects $[\mathrm{F}(1,18)=$ $2.72, \mathrm{NS}]$.
Both past response and past outcome, that is, whether the previous decision correctly or incorrectly predicted the location of the stimulus, significantly influenced decision-making of methamphetamine-dependent subjects during the two-choice prediction task. The mutual information function revealed that $\sim 2.8 \pm$ $0.8 \%$ of the current choice in methamphetamine-dependent subjects was predicted by the previous response (controls, : $3.5 \pm 0.8 \%$ ), whereas $19.6 \pm 3.6 \%$ of the current choice was predicted by a win-stay/lose-shift strategy (controls, $8.4 \pm 3.8 \%$ ). The ANOVA for the mutual information, that is, the degree to which the previous response predicted the current response, revealed no difference across trial blocks $\left[\mathrm{F}_{\mathrm{GG}}(2.6,47.9)=1.46\right.$, NS] but a trend across task conditions [mutual information: $\left.\mathrm{F}_{\mathrm{GG}}(1,18)=3.32, p=.085\right]$. In comparison, the degree to which a win-stay/lose-shift strategy predicted the current response did not differ across trial blocks $\left[\mathrm{F}_{\mathrm{GG}}(2.8,50.3)=0.89, \mathrm{NS}\right]$ but differed significantly across task conditions [win-stay/lose-shift mutual information: $\left.\mathrm{F}_{\mathrm{GG}}(1,18)=19.50, p<.01\right]$. There was a significant effect of group (methamphetamine-dependent subjects versus controls) on the degree to which winstay/lose-shift predicted the current response $[\mathrm{F}(1,18)=$ $4.91, p<.05]$. This indicates that, relative to controls, methamphetamine-dependent subjects used the winstay/lose-shift strategy more frequently when generating decision sequences during the two-choice prediction task. The analysis of the conditional win-stay/lose-shift probabilities (Figure 1) revealed that methamphetamine-dependent subjects (win-stay: $0.65 \pm 0.05$ ) were not more or less likely than controls (win-stay: $0.64 \pm$ $0.05)$ to use the win-stay strategy $\left[\mathrm{F}_{\mathrm{GG}}(1,18)=.178, \mathrm{NS}\right]$. In comparison, methamphetamine-dependent subjects selected more responses during the two-choice prediction task that were consistent with lose-shift (lose-shift:

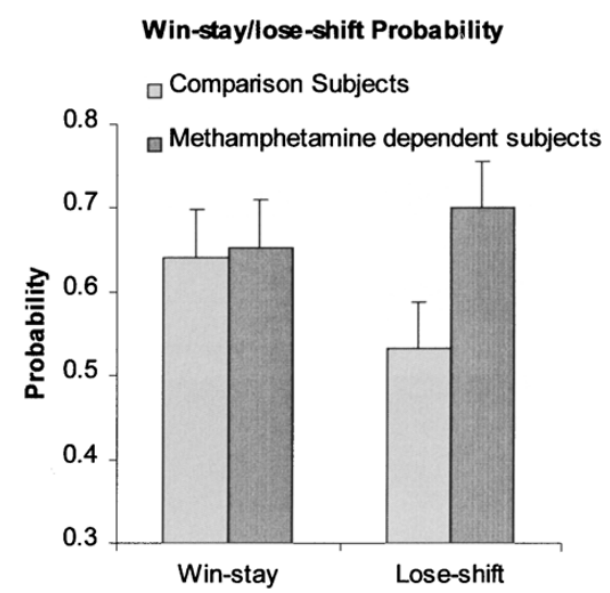

Figure 1. Probability of win-stay/lose-shift consistent responses during the two-choice prediction task in normal comparison and methamphetamine-dependent subjects. Error bars represent standard of mean. 
$0.70 \pm 0.05)$ than did controls (lose-shift: $0.53 \pm 0.05$ ) $\left[\mathrm{F}_{\mathrm{GG}}(1,18)=6.847, p<.01\right]$.

If the behavioral differences were due to the prolonged effect of methamphetamine, one would predict a lower degree of win-stay/lose-shift in subjects with longer duration of sobriety. Alternatively, if the behavioral differences are a result of extensive use of methamphetamine, one would predict an increased use of win-stay/lose-shift in subjects with a longer history of methamphetamine dependence. Therefore, the correlation between duration of sobriety or years of methamphetamine use and the probability of win-stay/loseshift consistent responses was obtained. As shown in Figure 2, subjects with a longer duration of sobriety showed less lose-shift consistent responses (Pearson's $\mathrm{r}=$ $-0.74, p<.05)$. In comparison, the duration of sobriety did not correlate with the number of win-stay consistent responses $(\mathrm{r}=-0.27$, NS). Therefore, the differences of response characteristics during the two-choice prediction task between methamphetamine-dependent and normal comparison subjects diminish with increased duration of sobriety. In contrast, duration of methamphetamine use (in years) did not correlate with the number of responses consistent with a lose-shift strategy $(\mathrm{r}=-0.25, \mathrm{NS})$ or with a win-stay strategy $(\mathrm{r}=$ $-0.08, \mathrm{NS})$.

\section{fMRI Measures}

Separate voxel-wise analyses for each group showed that both control subjects (Figures $3 \mathrm{~A}-\mathrm{C}$ ) and methamphetamine-dependent subjects (Figures 3D-F) exhibited task-related activation, that is, more echoplanar signal intensity during the two-choice prediction task relative to the two-choice response task, in prefrontal and parietal cortex. Similarly, functional regions of interest analysis showed that both controls and metham-

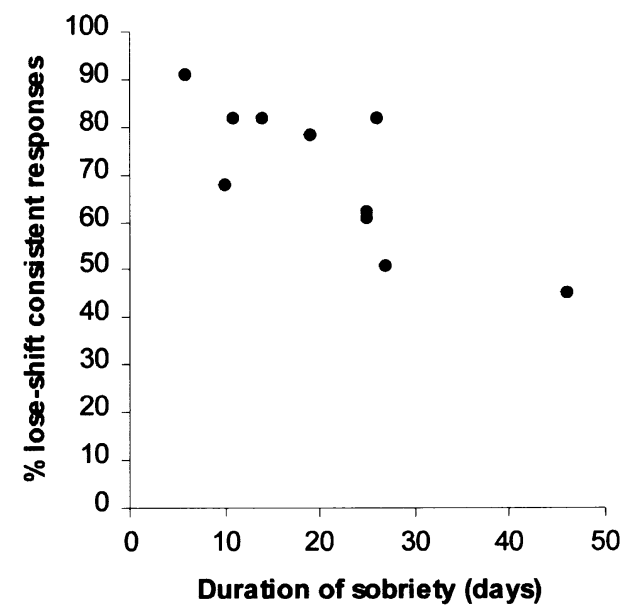

Figure 2. The percentage of lose-shift consistent responses in methamphetamine-dependent subjects declines with increasing duration of sobriety. phetamine-dependent subjects exhibited task-related activation in bilateral premotor, prefrontal, and parietal areas as well as in insula cortex (Table 2, Figure 4). The magnitude of task-related activation was significantly larger in controls relative to methamphetamine-depen-

\section{Comparison Subjects}

A

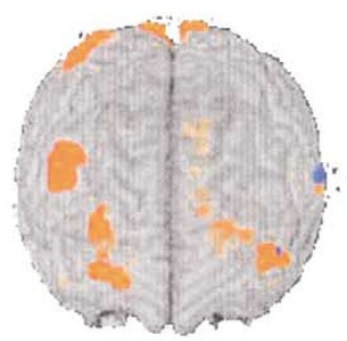

B

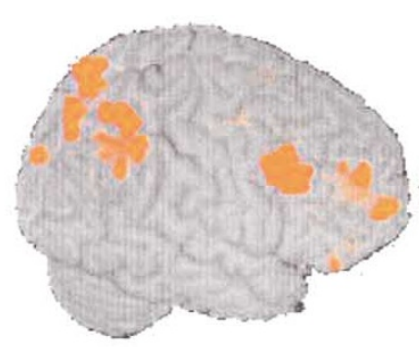

C

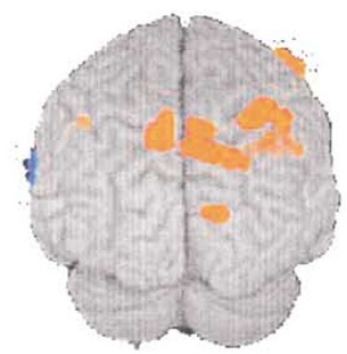

D

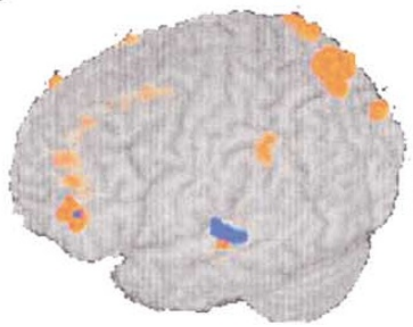

E

\section{Methamphetamine dependent Subjects}

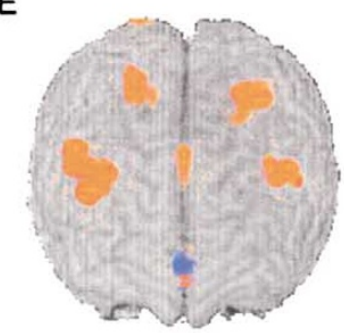

F

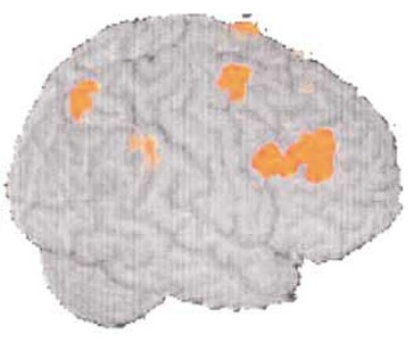

G

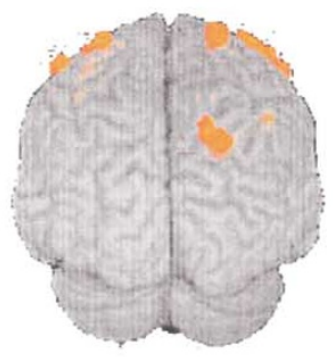

$\mathrm{H}$

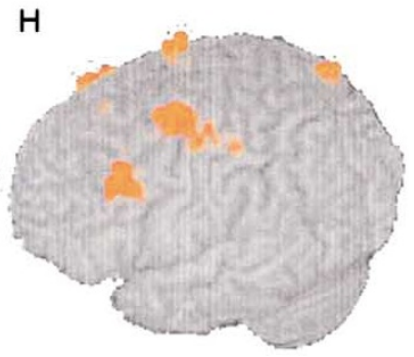

Figure 3. Volume-thresholded t-score renderings of the task-related activation in normal comparison (A-D) and methamphetamine-dependent subjects $(\mathbf{E}-\mathbf{H})$. 
Table 2. Location of Task-Related Activation Clusters for the Main Effect of Task in Both Methamphetamine-Dependent and Normal Comparison Subjects and the Group-by-Task Interaction

\begin{tabular}{lrrrlr}
\hline Volume $(\mu \mathbf{L})$ & $\mathbf{X}$ & $\mathbf{y}$ & $\mathbf{z}$ & \multicolumn{1}{c}{ L/R Comment } & BA \\
\hline Task effect & & & & & \\
540 & -29 & 1 & 56 & L middle frontal gyrus & 6 \\
837 & 21 & -3 & 60 & R middle frontal gyrus & 6 \\
351 & 47 & 16 & 12 & R inferior frontal gyrus & 44 \\
378 & -41 & 44 & 6 & L middle frontal gyrus & 46 \\
756 & -34 & 18 & 4 & L insula & 13 \\
1566 & 35 & 20 & 6 & R insula & 13 \\
405 & -12 & -70 & 56 & L superior parietal lobule & 7 \\
756 & 36 & -54 & 54 & R superior parietal lobule & 7 \\
756 & 4 & -59 & 43 & L/R precuneus & 7 \\
1134 & 12 & -74 & 50 & R precuneus & 7 \\
405 & -44 & 8 & -19 & L superior temporal gyrus & 38 \\
Group-by-task interaction & & & & & \\
756 & -24 & 40 & 14 & L middle frontal gyrus & $9 / 10$ \\
432 & -4 & 51 & 17 & L/R medial frontal gyrus & 10 \\
675 & 19 & 55 & -16 & R orbitofrontal gyrus & 11 \\
\hline
\end{tabular}

The cluster volume is given in microliters and the center of mass of the cluster is given in Talairach coordinates. Abbreviations: $\mathrm{L}=$ Left, $\mathrm{R}=$ Right.

dent subjects in the right inferior frontal gyrus (BA 44) $[\mathrm{t}(18)=2.01, p<.05]$ and the left middle frontal gyrus (BA 46) $[\mathrm{t}(18)=2.004, p<.05]$. As shown in Figure 5 and Table 2, the interaction effect between task and group of the mixed model ANOVA revealed that controls showed an increase in echoplanar signal intensity dur-

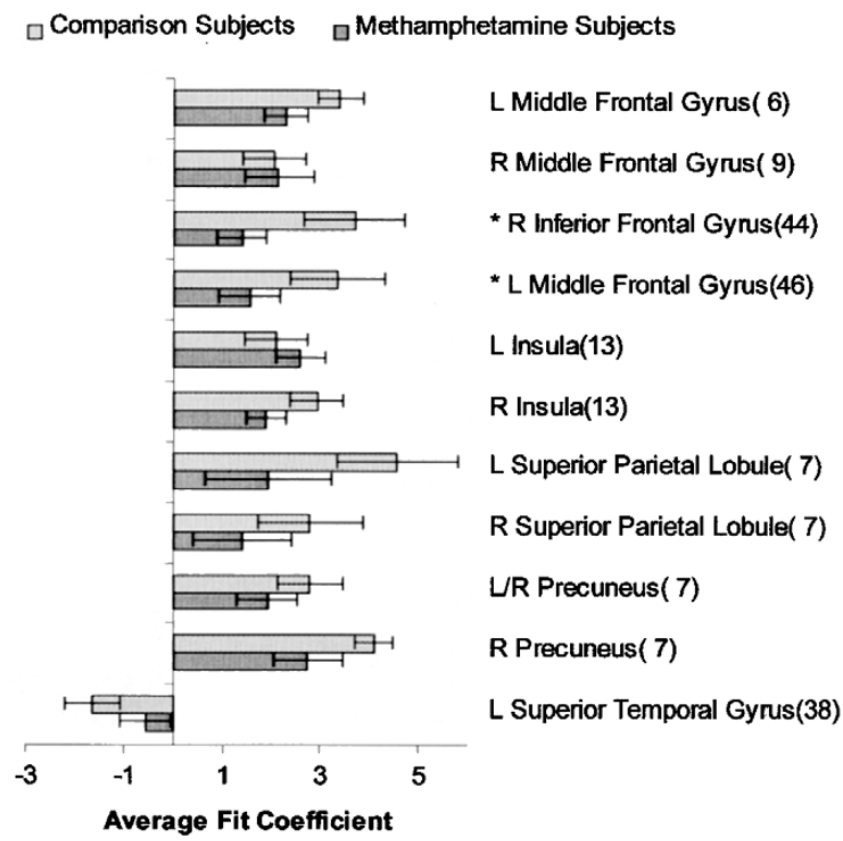

Figure 4. The ROI-specific average fit coefficient and SEM for both methamphetamine-dependent and normal comparison subjects for the functional areas of interest, as determined by the main effect of task. Asterisks indicate significant differences in activation between methamphetamine-dependent and normal comparison subjects. Brodman areas are shown in parentheses. Error bars represent SEM. ing the two-choice prediction task relative to the twochoice response task in the left middle frontal gyrus (BA9/10), in left and right medial frontal gyrus (BA 10) and right orbitofrontal gyrus (BA 11). In contrast, methamphetamine-dependent subjects showed no change in task-related activation (left BA 9/10) or showed a decrease in echoplanar signal intensity during the twochoice prediction task relative to the two-choice response task (bilateral BA 10 and right BA 11) (Figure 5, right). These areas correspond to areas of the dorsolateral and anterior orbitofrontal cortex ( $\mathrm{O}^{\prime}$ Doherty et al. 2001). This interaction between task and group supports the hypothesis that methamphetamine-dependent subjects relative to controls failed to activate orbitofrontal parts for the prefrontal cortex during the two-choice prediction task, relative to the two-choice response task.

ROI specific fit coefficients were used in a stepwise regression analysis to predict the percentage of loseshift consistent responses, the duration of sobriety, and years of methamphetamine use, in order to relate taskrelated activation to clinical variables. Relative lower level of activation in the left insula (BA 13, $-0.14 \pm$ $0.02, \mathrm{t}=5.89, p<.05)$ and relative higher level of activation in the right middle frontal gyrus (BA 9, $0.07 \pm$ $0.02, \mathrm{t}=3.11, p<.05$ ) predicted a higher percentage of lose-shift consistent responses in methamphetaminedependent subjects $\left[\mathrm{F}(2,9)=18.85, p<.01, \mathrm{r}^{2}=0.8\right]$. In comparing this relationship to the degree of task-related activation in control subjects, this finding is consistent with the observation that methamphetamine-dependent subjects showed slightly but nonsignificantly higher activation in the left insula cortex. A relative higher level of task-related activation in the left middle frontal gyrus (BA 6, $4.35 \pm 1.1, \mathrm{t}=3.7, p<.05)$ and a relative 


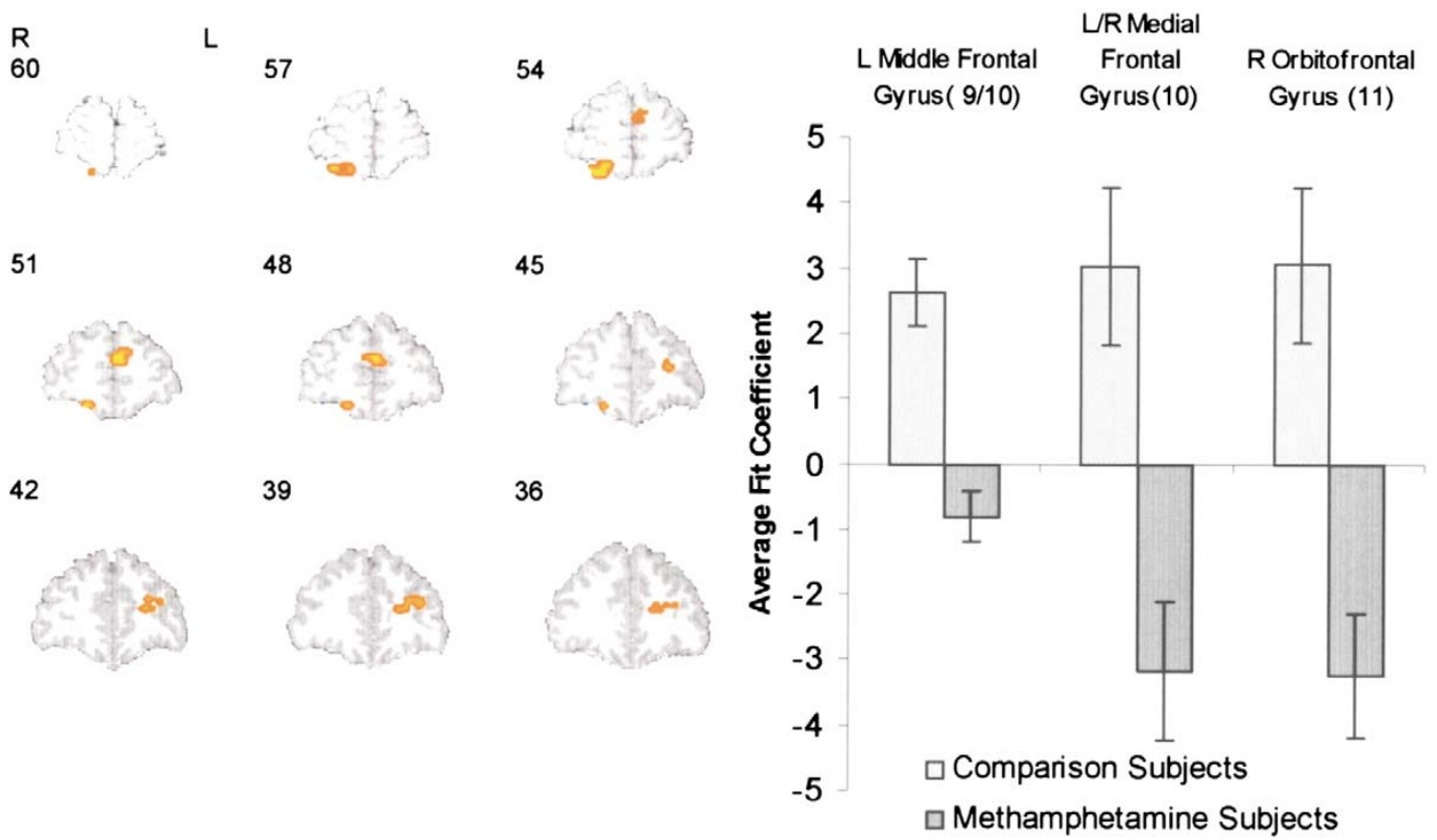

Figure 5. Task-by-group interaction. Left) Volume-threshold F-map of interaction effect. Numbers correspond to Talairach y-coordinate; cluster centers are shown in Table 2. Right) ROI-specific average fit coefficient and SEM for both methamphetamine-dependent and normal comparison subjects in the left middle frontal gyrus, bilateral medial frontal gyrus, and right orbitofrontal gyrus. Brodmann areas are shown in parenthesis.

lower level of task-related activation in the left superior temporal gyrus (BA 38, $-4.80 \pm 1.8, \mathrm{t}=2.6, p<.05$ ) predicted a longer period of sobriety in methamphetamine-dependent subjects $\left[\mathrm{F}(2,9)=6.9, p<.05, \mathrm{r}^{2}=\right.$ 0.57]. Finally, subjects with a larger decrease in the orbitofrontal gyrus (BA 11, $-1.54 \pm 0.7, \mathrm{t}=2.4, p<.05$ ), that is, the area of the interaction effect between task and group, had a longer duration of methamphetamine use $\left[\mathrm{F}(1,9)=5.6, p<.05, \mathrm{r}^{2}=0.34\right]$. These results show that some areas of activation in the right and left prefrontal cortex, insula, and superior temporal gyrus can be related to the response characteristics during the two-choice prediction task and the clinical characteristics of the methamphetamine-dependent subjects. Moreover, the lack of task-related activation in the orbitofrontal cortex is related to the duration of methamphetamine use.

\section{DISCUSSION}

This investigation yielded five main results. First, methamphetamine-dependent subjects relative to normal comparison subjects relied more on an outcome-dependent strategy (win-stay/lose-shift) during the twochoice prediction task than did normal comparison subjects. Second, the degree of the behavioral differences between these groups diminished with increased dura- tion of sobriety. Third, similar to normal comparison subjects, methamphetamine-dependent subjects exhibited task-related activation in bilateral prefrontal, parietal, and insula cortex; however, they showed less inferior prefrontal task-related activation. Fourth, methamphetamine-dependent subjects failed to show task-related activation in left prefrontal cortex (BA 9/10), bilateral ventromedial prefrontal cortex (BA 10), and right orbitofrontal cortex (BA 11). Fifth, the activation in the right orbitofrontal cortex predicted the duration of methamphetamine use.

The increase in win-stay/lose-shift consistent responses during the two-choice prediction task in methamphetamine-dependent subjects supports the general hypothesis that these subjects, even in situations without a priori advantageous or disadvantageous response bias, are more driven by the immediately preceding outcome (win or lose) than are normal comparison subjects. Thus, the increased use of an outcome-contingent response strategy by methamphetamine-dependent subjects is consistent with other behavioral observations in decision-making studies with substance-dependent subjects. First, in a decision-making task involving a choice between a smaller immediate reward and a larger but delayed reward, these subjects selected responses using short interdecision intervals that were associated with more immediate reward (Allen et al. 1998). Second, using a task that assesses decision-mak- 
ing in the presence of varying likelihoods of reward and varying reward magnitude, amphetamine-dependent subjects took longer to make a decision and used suboptimal decisions strategies (Rogers et al. 1999a). Third, using different variations of the gambling task (Petry et al. 1998), heroin- and cocaine-dependent subjects were found to select responses that were associated with greater immediate gains but overall net losses (Bartzokis et al. 2000). Finally, polysubstance abusers were found to select more disadvantageously when choosing between small rewards but consistent gains versus high rewards associated with significant losses (Grant et al. 2000). When separating the win-stay/loseshift strategy into its components, it appears the increase in the mutual information is primarily due to the increased frequency of lose-shift responses. This finding supports the hypothesis that methamphetaminedependent subjects are more sensitive to immediately preceding incorrect predictions than controls. The observation that the degree to which these response sequences in humans decreased with duration of sobriety suggests that the increased sensitivity to punished responses is a temporary behavioral change; this needs to be further evaluated in methamphetamine-dependent subjects at different stages of sobriety.

Some investigators have pointed out that suboptimal response selection may not only result as a function of dysregulated stimulus-reward association but may simply be due to an increase in response perseveration (Rolls 2000), which may relate to dysfunctions of the dorsolateral prefrontal cortex (Jahanshahi and Dirnberger 1999). The degree to which the previous response predicted the current response as measured by the mutual information did not differ between methamphetamine-dependent and normal comparison subjects. Thus, methamphetamine-dependent subjects did not show simple response perseveration during the twochoice prediction task. Moreover, both methamphetamine-dependent and normal comparison subjects showed similar magnitude of task-related right prefrontal (BA 9) activation, an area that is critical for executive functioning. Nevertheless, future studies need to examine the influence of reward magnitude in methamphetamine-dependent subjects to determine whether the behavioral and neuroimaging differences are due to a dysfunctional influence of the association between stimulus and reward on decision-making or whether the decision-making is a result of stimulus perseveration.

The differences in task-related activation between methamphetamine-dependent and normal comparison subjects in both dorsolateral prefrontal (BA 9), medial prefrontal (BA 10), and orbitofrontal cortex (BA 11) are consistent with previous studies, which show that these areas are critical for decision-making (Elliott et al. 1997; Rogers et al. 1999b, 2000), task-related reward contingencies (Elliott et al. 1999), and violation of expectations
(Nobre et al. 1999). Several cellular and neuroimaging studies support the hypothesis that decision-making involves prefrontal, parietal, and cingulate cortex neural network. First, self-generated, as opposed to cue-generated, actions involve the activation of dorsolateral prefrontal cortex and anterior cingulate (Frith et al. 1991). Second, activity associated with anticipating a gaze response in a decision-making task was found in individual neurons in BA 9 and 46, that is, the dorsolateral prefrontal cortex (Kim and Shadlen 1999). Third, activity of single neurons in the lateral parietal area was associated with selecting a response in a decision-making task in a reinforcement and reward-related manner (Platt and Glimcher 1999). Fourth, explicit hypothesis testing in a two-choice decision-making task was associated with a significant activation of the left anterior cingulate, right precuneus, right thalamus, left inferior frontal gyrus, and cerebellum (Elliott and Dolan 1998). Fifth, using fMRI, both right ventromedial and dorsolateral prefrontal cortex were activated in a two-choice guessing task relative to a choice reporting task (Elliott et al. 1999). Sixth, using a gamble task, Rogers et al. (1999b) found a discrete increase in blood flow in right inferior and orbital prefrontal cortex (BA 10,11) and in the anterior portion of the inferior frontal gyrus (BA 47).

The differences in activation patterns between methamphetamine-dependent and normal comparison subjects support the hypothesis that substance-dependent subjects show a dysregulation of the striato-thalamo-orbitofrontal circuit (Volkow and Fowler 2000), which is critical for decision-making. After damage to the orbitofrontal cortex, humans develop a defect in real-life decision-making, which contrasts with otherwise normal intellectual functions (Damasio et al. 1990). Specifically, these subjects are guided by the immediate prospects but seem less affected by future consequences of their actions (Bechara et al. 1994). It has been suggested that this region is an important neural structure in decisionmaking and functions independently of working memory load, which is mediated in the dorsolateral prefrontal cortex. People with chronic amphetamine dependence and patients with focal lesions of the orbitofrontal cortex showed similar choice patterns in an experimental decision-making task (Rogers et al. 1999a). These subjects showed significant increases in the time needed to make their decisions, which was particularly evident in the presence of unclear predictive information. Moreover, the stimulant-abusing subjects relative to controls consistently selected a response that was less optimal in the presence of predictive information (Grant et al. 2000). Finally, chronic amphetamine abusers were found to be impaired on an extradimensional shift task, a core component of set-shifting (Ornstein et al. 2000). These results lend support to the basic hypothesis that stimulant-dependent subjects exhibit fundamental cognitive deficits that are consistent 
with both ventromedial and dorsolateral prefrontal dysfunction. The differences in dorsolateral prefrontal cortex activation is consistent with the increased frequency of responses contingent on the immediately preceding outcome (win-stay/lose-shift) rather than according to an ongoing strategy that is kept in working memory. The activation pattern differences are consistent with reports that chronic methamphetamine use results in changes of biological markers of neuronal integration in cortical areas (Ernst et al. 2000) and changes of dopamine transporter density in subcortical areas (McCann et al. 1998).

This investigation has several limitations. First, the number of methamphetamine-dependent subjects is relatively small for behavioral studies. Although the effect sizes between methamphetamine-dependent subjects and controls for the two task types were large, future investigations with more subjects will facilitate further testing of the relationship between stimulant use and outcome-driven response strategies. Second, the current sample consists solely of male subjects; thus, female controls and methamphetamine-dependent subjects will need to be investigated to determine whether this finding is consistent in both genders. Third, three of the 10 methamphetamine dependent subjects were comorbid for cannabis abuse. Although preliminary analysis of the functional ROIs did not reveal significant differences in task-related activation between these and the other methamphetamine-dependent subjects (data not shown), the influence of cannabis on these results will need to be further examined in a larger group. Fourth, the functional neuroimages of all subjects were obtained after a period of $6-46$ days of abstinence, which does not permit conclusions about the long-term effect of methamphetamine on the functional status of the ventromedial prefrontal cortex. Finally, it will be important to see if any of the fMRI results are seen before the development of amphetamine dependence.

Methamphetamine-dependent subjects relative to normal comparison subjects show decision-making behavior that is more driven by the immediate outcome and show less task-related activation of both dorsolateral and orbitofrontal cortex. The reduced activation of dorsolateral and orbitofrontal cortex in methamphetamine dependent subjects was not related to duration of sobriety. Thus, chronic use of stimulants may have induced long-lasting changes consistent with abnormal orbitofrontal activity (Volkow et al. 1993).

\section{ACKNOWLEDGMENTS}

We would like to acknowledge the invaluable help of Rick Buxton and Eric Wong in supporting the fMRI experiments. This work was supported by grants from NIMH (MH R37-
42228 to DLB; R21DA13186 to MPP), NARSAD (MPP), and support from the Veterans Administration via a Merit Award (MPP) and a VISN 22 MIRECC.

\section{REFERENCES7}

Allen TJ, Moeller FG, Rhoades HM, Cherek DR (1998): Impulsivity and history of drug dependence. Drug Alcohol Depend. 50:137-145

American Psychiatric Association (1994): Diagnostic and Statistical Manual of Mental Disorders, 4th edition (DSM-IV). Washington DC, American Psychiatric Press

Bartzokis G, Lu PH, Beckson M, Rapoport R, Grant S, Wiseman EJ, London ED (2000): Abstinence from cocaine reduces high-risk responses on a gambling task. Neuropsychopharmacology 22:102-103

Bechara A, Damasio AR, Damasio H, Anderson SW (1994): Insensitivity to future consequences following damage to human prefrontal cortex. Cognition 50:7-15

Cohen MS (1997): Parametric analysis of fMRI data using linear systems methods. Neuroimage 6:93-103

Cox RW (1996): AFNI: Software for analysis and visualization of functional magnetic resonance neuroimages. Computers and Biomedical Research 29:162-173

Damasio AR, Tranel D, Damasio H (1990): Individuals with sociopathic behavior caused by frontal damage fail to respond autonomically to social stimuli. Behav Brain Res 41:81-94

Elliott R, Dolan RJ (1998): Activation of different anterior cingulate foci in association with hypothesis testing and response selection. Neuroimage 8:17-29

Elliott R, Frith CD, Dolan RJ (1997): Differential neural response to positive and negative feedback in planning and guessing tasks. Neuropsychologia 35:1395-1404

Elliott R, Rees G, Dolan RJ (1999): Ventromedial prefrontal cortex mediates guessing. Neuropsychologia 37:403411

Ernst T, Chang L, Leonido-Yee M, Speck O (2000): Evidence for long-term neurotoxicity associated with methamphetamine abuse: A $1 \mathrm{H}$ MRS study. Neurology 54: 1344-1349

Forman SD, Cohen JD, Fitzgerald M, Eddy WF, Mintun MA, Noll DC (1995): Improved assessment of significant activation in functional magnetic resonance imaging (fMRI): Use of a cluster-size threshold. Magn Reson Med 33:636-647

Frith CD, Friston K, Liddle PF, Frackowiak RS (1991): Willed action and the prefrontal cortex in man: A study with PET. Proc R Soc Lond B Biol Sci 244:241-246

Grant S, Contoreggi C, London ED (2000): Drug abusers show impaired performance in a laboratory test of decision making. Neuropsychologia 38:1180-1187

Green L, Myerson J,and Ostaszewski P (1999): Amount of reward has opposite effects on the discounting of delayed and probabilistic outcomes. J Exp Psychol Learn Mem Cogn 25:418-427

Herzel H, Grosse I (1997): Correlations in DNA sequences: The role of protein coding segments. Physical Review E 55:800-810 
Jahanshahi M, Dirnberger G (1999): The left dorsolateral prefrontal cortex and random generation of responses: Studies with transcranial magnetic stimulation. Neuropsychologia 37:181-190

Kim JN, Shadlen MN (1999): Neural correlates of a decision in the dorsolateral prefrontal cortex of the macaque. Nat Neurosci 2:176-185

Lancaster JL, Woldorff MG, Parsons LM, Liotti M, Freitas CS, Rainey L, Kochunov PV, Nickerson D, Mikiten SA, Fox PT (2000): Automated Talairach atlas labels for functional brain mapping. Hum Brain Mapp 10:120-131

London ED, Ernst M, Grant S, Bonson K, Weinstein A (2000): Orbitofrontal cortex and human drug abuse: Functional imaging. Cereb Cortex. 10:334-342

McCann UD, Wong DF, Yokoi F, Villemagne V, Dannals RF, Ricaurte GA (1998): Reduced striatal dopamine transporter density in abstinent methamphetamine and methcathinone users: Evidence from positron emission tomography studies with [11C]WIN-35,428. J Neurosci 18:8417-8422

Monterosso J, Ainslie G (1999): Beyond discounting: Possible experimental models of impulse control. Psychopharmacology (Berl.) 146:339-347

Nobre AC, Coull JT, Frith CD, Mesulam MM (1999): Orbitofrontal cortex is activated during breaches of expectation in tasks of visual attention. Nat Neurosci 2:11-12

Norusis MJ (1990): SPSS Base System User's Guide. Chicago, SPSS Inc.

O'Doherty J, Rolls ET, Francis S, Bowtell R, McGlone F, Kobal G, Renner B, Ahne G (2000): Sensory-specific satiety-related olfactory activation of the human orbitofrontal cortex. Neuroreport 11:893-897

O'Doherty J, Kringelbach ML, Hornak J, Andrews C, Rolls ET (2001): Abstract reward and punishment representations in the human orbitofrontal cortex. Nat Neurosci 4:95-102

Ornstein TJ, Iddon JL, Baldacchino AM, Sahakian BJ, London M, Everitt BJ, Robbins TW (2000): Profiles of cognitive dysfunction in chronic amphetamine and heroin abusers. Neuropsychopharmacology 23:113-126

Paulus MP (1997): Long-range interactions in sequences of human behavior. Physical Review E 55:3249-3256

Paulus MP, Geyer MA, Braff DL (1994): The assessment of sequential response organization in schizophrenic and control subjects. Prog.Neuropsychopharmacol Biol Psychiatry 18:1169-1185

Paulus MP, Geyer MA, Braff DL (1996): Use of methods from chaos theory to quantify a fundamental dysfunction in the behavioral organization of schizophrenic patients. Am J Psychiatry 153:714-717

Paulus MP, Hozack N, Zauscher B, McDowell JE, Frank L, Brown GG, Braff DL (2001): Prefrontal, parietal, and temporal cortex networks underlie decision-making in the presence of uncertainty. Neuroimage 13:91-100

Petry NM, Bickel WK, Arnett M (1998): Shortened time horizons and insensitivity to future consequences in heroin addicts. Addiction 93:729-738

Platt ML, Glimcher PW (1999): Neural correlates of decision variables in parietal cortex. Nature 400:233-238

Rahman S, Sahakian BJ, Hodges JR, Rogers RD, Robbins TW (1999): Specific cognitive deficits in mild frontal variant frontotemporal dementia. Brain 122:1469-1493

Rogers RD, Everitt BJ, Baldacchino A, Blackshaw AJ, Swainson R, Wynne K, Baker NB, Hunter J, Carthy T, Booker E, London M, Deakin JF, Sahakian BJ, Robbins TW (1999a): Dissociable deficits in the decision-making cognition of chronic amphetamine abusers, opiate abusers, patients with focal damage to prefrontal cortex, and tryptophan-depleted normal volunteers: Evidence for monoaminergic mechanisms. Neuropsychopharmacology 20:322-339

Rogers RD, Owen AM, Middleton HC, Williams EJ, Pickard JD, Sahakian BJ, Robbins TW (1999b): Choosing between small, likely rewards and large, unlikely rewards activates inferior and orbital prefrontal cortex. J Neurosci. 19:9029-9038

Rogers RD, Andrews TC, Grasby PM, Brooks DJ, Robbins TW (2000): Contrasting cortical and subcortical activations produced by attentional-set shifting and reversal learning in humans. J Cogn Neurosci 12:142-162

Rolls ET (2000): The orbitofrontal cortex and reward. Cereb Cortex. 10:284-294

Seale DA, Rapoport A (1997): Sequential decision making with relative ranks: An experimental investigation of the "secretary problem.". Organizational Behavior \& Human Decision Processes 69:221-236

Spitzer RL, Williams JB, Gibbon M, First MB (1992): The Structured Clinical Interview for DSM-III-R (SCID): I. History, rationale, and description. Arch Gen Psychiatry 49:624-629

Talairach J, Tournoux P (1988): Co-Planar Stereotaxic Atlas of the Human Brain: A 3-Dimensional Proportional System, an Approach to Cerebral Imaging. New York, Thieme Medical Publishers

Tversky A, Sattath S, Slovic P (1988): Contingent weighting in judgment and choice. Psychol Rev 95:371-384

Volkow ND, Fowler JS (2000): Addiction, a disease of compulsion and drive: Involvement of the orbitofrontal cortex. Cereb Cortex. 10:318-325

Volkow ND, Fowler JS, Wang GJ, Hitzemann R, Logan J, Schlyer DJ, Dewey SL, Wolf AP (1993): Decreased dopamine D2 receptor availability is associated with reduced frontal metabolism in cocaine abusers. Synapse 14:169177 